\title{
Continuum limits of bistable spring models of carbon nanotube arrays accounting for material damage
}

\author{
T. Blesgen, F. Fraternalił J. R. Raney; A. Amendola, C. Daraio
}

December 13, 2011

\begin{abstract}
Using chains of bistable springs, a model is derived to investigate the plastic behavior of carbon nanotube arrays with damage. We study the preconditioning effect due to the loading history by computing analytically the stress-strain pattern corresponding to a fatigue-type damage of the structure. We identify the convergence of the discrete response to the limiting case of infinitely many springs, both analytically in the framework of Gamma-convergence, as well as numerically.
\end{abstract}

\section{Keywords}

Carbon nanotube arrays; bistable springs; multiscale behavior; Mullins effect; permanent deformation.

\section{Introduction}

Because of their interesting combination of properties, including high strength, low density, and high electrical and thermal conductivities, carbon nanotubes (CNTs) have been of great interest as nanoscale elements in a variety of applications, [1]. Aligned arrays of CNTs can be readily synthesized to form foam-like materials that combine low density with a desirable dissipative response, $[6,10]$.

\footnotetext{
* Max Planck Institute for Mathematics in the Sciences, Inselstraße 22, D-04103 Leipzig, Germany, email: blesgen@mis.mpg.de

${ }^{\dagger}$ Department of Civil Engineering, University of Salerno, 84084 Fisciano(SA), Italy, email: f.fraternali@unisa.it

${ }^{\ddagger}$ Engineering and Applied Science, California Institute of Technology, Pasadena, CA 91125, USA, email: raney@caltech.edu

${ }^{\S}$ Department of Civil Engineering, University of Salerno, 84084 Fisciano(SA), Italy, email: adamendola@gmail.com

『 Engineering and Applied Science, California Institute of Technology, Pasadena, CA 91125, USA, email: daraio@caltech.edu
} 
There are a few interesting structural and mechanical features of these materials: First, the thermal chemical vapor deposition process that is typically used to synthesize the arrays [6] results in a gradient in physical properties (such as density) along the height of the structure. This leads to strain localization during compression, with the majority of the structure remaining undeformed while increasing strain results in the sequential addition of highly localized buckles $[18,11]$. Second, there is a large amount of strain recovery after compression (typical of the CNT arrays that we study, which are synthesized using a vapor phase catalyst, [6], but not for all types of CNT arrays that are synthesized differently, $[18,3])$. Third, the stress-strain response of the material is hysteretic, with different loading and unloading paths. It has been noted in the past that repeated compressive cycles result in a hysteresis of decreasing area and decreasing stress at any given strain for the first few cycles, [6]. This effect, sometimes referred to as preconditioning, ceases after the initial few cycles, resulting in a hysteresis of constant area for loading cycles thereafter. Finally, we observe that this preconditioning effect is dependent on the maximum strain reached. When the maximum strain of all previous loading cycles is exceeded, the stress response obtained at these elevated strains is that of the un-preconditioned material, as if it had never been compressed previously, [12].

The features we have enumerated for the foam-like response of CNT arrays are analogous to those observed in other soft materials such as filled rubbers. In the context of rubbers, this response is associated with what is termed the Mullins effect, $[13,8]$. Capturing these features simultaneously in models has proven difficult in the past for other materials, with frequent use of simplifying assumptions that only allow models to match some of the experimental observations (see, e.g., the idealized Mullins effect modeled in [7]).

We show in the present work that a suitable generalization of the mesoscopic mass-spring model of CNT structures recently proposed by [9] is able to handle the material damage due to preconditioning and permanent deformation within an effective one-dimensional framework. We introduce preconditioning-induced material damage by setting to zero the stiffness of a suitable percentage of the bistable springs that describe the response of the material at the microscopic scale. This allows us to model permanent axial deformation of the structure through the irreversible 'annihilation' of the springs with zero stiffness.

\section{Multiscale mass-spring models of CNT arrays}

\subsection{Bistable spring model at the microscopic scale}

We model an infinitesimal portion of a CNT foam through the bistable spring model described in [9], which we hereafter briefly summarize. We assume that such a portion of the foam can be described as a chain of $N+1$ lumped masses

$m^{0}, \ldots, m^{N}$, with $m^{0}$ clamped at the bottom of the chain. The adjacent masses are connected to each other through bistable springs characterized by the axial 
strains

$$
\varepsilon^{i}=\varepsilon^{i}\left(u_{N}\right)=\frac{u_{N}^{i-1}-u_{N}^{i}}{h_{N}}, \quad i=1, \ldots, N
$$

where $u_{N}^{i}$ is the axial displacement of the mass $m^{i}$ (positive upward), $h_{N}:=$ $L / N$ is the equal spacing between the masses, and $u_{N}:=\left\{u_{N}^{0}, \ldots, u_{N}^{N}\right\}$. The potential $V^{i}$ and stress $\sigma^{i}$ vs strain $\varepsilon^{i}$ laws of the generic spring are

$$
\begin{gathered}
V^{i}\left(\varepsilon^{i}\right):= \begin{cases}V_{a}^{i}\left(\varepsilon^{i}\right):=-k_{0}^{i}\left[\varepsilon^{i}+\ln \left(1-\varepsilon^{i}\right)\right], & \varepsilon^{i}<\varepsilon_{a}^{i}, \\
V_{b}^{i}\left(\varepsilon^{i}\right):=c_{1}+\sigma_{a}^{i} \varepsilon^{i}+\frac{1}{2} k_{b}^{i}\left(\varepsilon^{i}-\varepsilon_{a}^{i}\right)^{2}, & \varepsilon_{a}^{i} \leq \varepsilon^{i} \leq \bar{\varepsilon}_{c}^{i}, \\
V_{c}^{i}\left(\varepsilon^{i}\right):=c_{2}-k_{c}^{i}\left[\varepsilon^{i}-\varepsilon_{*}^{i}+\ln \left(1-\left(\varepsilon^{i}-\varepsilon_{*}^{i}\right)\right)\right], & \bar{\varepsilon}_{c}^{i}<\varepsilon^{i},\end{cases} \\
\sigma^{i}\left(\varepsilon^{i}\right)=V^{i^{\prime}}\left(\varepsilon^{i}\right)= \begin{cases}k_{0}^{i} \frac{\varepsilon^{i}}{1-\varepsilon^{i}}, & \varepsilon^{i}<\varepsilon_{a}^{i}, \\
\sigma_{a}^{i}+k_{b}^{i}\left(\varepsilon^{i}-\varepsilon_{a}^{i}\right), & \varepsilon_{a}^{i} \leq \varepsilon^{i} \leq \bar{\varepsilon}_{c}^{i}, \\
\frac{k_{c}^{i}\left(\varepsilon^{i}-\varepsilon_{*}^{i}\right)}{1-\left(\varepsilon^{i}-\varepsilon_{*}^{i}\right)}, & \bar{\varepsilon}_{c}^{i}<\varepsilon^{i}\end{cases}
\end{gathered}
$$

where $k_{0}^{i}>0, k_{b}^{i}<0, k_{c}^{i}>0, \varepsilon_{a}^{i}>0$ and $\varepsilon_{c}^{i} \geq \varepsilon_{a}^{i}$ are constitutive parameters (five independent parameters); the constants $c_{1}<0$ and $c_{2}>0$ are such that $V_{a}^{i}\left(\varepsilon_{a}^{i}\right)=V_{b}^{i}\left(\varepsilon_{a}^{i}\right), V_{b}^{i}\left(\bar{\varepsilon}_{c}^{i}\right)=V_{c}^{i}\left(\bar{\varepsilon}_{c}^{i}\right)$; and it results (compare with Fig. 2 and Fig. 3 of [9] for the notation)

$$
\varepsilon_{*}^{i}=\varepsilon_{c}^{i}-\frac{\sigma_{a}^{i}}{k_{c}^{i}+\sigma_{a}^{i}}, \quad \bar{\varepsilon}_{c}^{i}=\frac{\varepsilon_{c}^{i}\left(k_{c}^{i}+\sigma_{a}^{i}\right)}{k_{c}^{i}+\sigma_{c}^{i}}+\frac{\left(\sigma_{c}^{i}-\sigma_{a}^{i}\right)\left(k_{c}^{i}+\varepsilon_{c}^{i} k_{c}^{i}+\varepsilon_{c}^{i} \sigma_{a}^{i}\right)}{\left(k_{c}^{i}+\sigma_{a}^{i}\right)\left(k_{c}^{i}+\sigma_{c}^{i}\right)}
$$

with $\sigma_{a}^{i}=k_{0}^{i} \frac{\varepsilon_{a}^{i}}{1-\varepsilon_{a}^{i}}, \sigma_{c}^{i}=\sigma_{a}^{i}+k_{b}^{i}\left(\bar{\varepsilon}_{c}^{i}-\varepsilon_{a}^{i}\right)$.

\section{$2.2 \quad$ Plasticity and damage}

At the microscopic scale, the bistable springs introduced above permit a dynamic switching process between the phases (a) and (c), cf. [14, 15]. As in [15], we name a response of the material plastic, if the strain $\varepsilon^{i}$ of a single spring exceeds the threshold $\varepsilon_{a}^{i}$. For a chain of $N$ springs, this can be characterized by the occurrence of loading and unloading stress plateaux.

Within the current section, we rescale for simplicity $L$ to unity; name (b) the unstable phase; and regard a mesoscopic element of a CNT array as the limit $N \rightarrow \infty$ of a series of $N$ microscopic springs.

Let $m$ denote the number of hysteresis cycles that have been applied to the material during the previous loading history (up to different maximum strains). We assume that such a loading path has severely weakened the stiffness of $(1-\beta(m)) N$ microscopic springs, for given $0<\beta(m) \leq 1$, and that at the current time it holds for all $m \in \mathbb{N}$

$$
\begin{array}{ll}
(A 1) & k_{c}^{i}=k_{0}^{i} \quad \text { for all } i \in \mathbb{N} \\
(A 2) & k_{0}^{1}=k_{0}^{2}=\ldots=k_{0}^{\lfloor\beta N\rfloor}=k_{0}, \quad k_{0}^{\lfloor\beta N+1\rfloor}=\ldots=k_{0}^{N}=\delta \\
(A 3) & \varepsilon_{*}^{i}=\varepsilon_{*} \quad \text { for } i=1, \ldots, \beta N \\
(A 4) & \varepsilon_{a}^{i}=\bar{\varepsilon}_{c}^{i} \quad \text { for } i=\beta N+1, \ldots, N .
\end{array}
$$


Condition (A1) stipulates the symmetry of the microscopic springs. (A2) states that the springs $\lfloor\beta N+1\rfloor$ to $N$ have stiffness $k_{0}^{i}=\delta$, where $\delta>0$ is a small constant (damaged springs). We name undamaged those springs with stiffness constant $k_{0}$ (springs 1 to $\beta N$ ). For the present analysis we also require a certain smallness condition on $\varepsilon_{a}^{i}$ and $\bar{\varepsilon}_{c}^{i}$ relating to strong pinning that disappears for $N \rightarrow \infty$, see [14].

With $V^{i}$ given by (2), the mechanical energy of the structure is

$$
E_{N}\left(u_{N}\right):=\frac{1}{N} \sum_{i=1}^{N} V^{i}\left(\varepsilon^{i}\left(u_{N}\right)\right)
$$

Let $\sigma$ be the given total stress. The mesoscopic average strain is $\operatorname{simply} \varepsilon\left(u_{N}\right):=$ $\frac{1}{N} \sum_{i=1}^{N} \varepsilon^{i}\left(u_{N}\right)$, where $\varepsilon^{i}$ denotes the strain associated with the $i$-th spring. Following an original idea of [15], we model plasticity by the gradient flow equations

$$
\nu \dot{\varepsilon}^{i}\left(u_{N}\right)=-\frac{\partial \Phi_{N}}{\partial \varepsilon^{i}}\left(\varepsilon^{1}\left(u_{N}\right), \ldots, \varepsilon^{N}\left(u_{N}\right)\right)
$$

with the total energy

$$
\Phi_{N}\left(\varepsilon^{1}, \ldots, \varepsilon^{N}\right):=\frac{1}{N} \sum_{i=1}^{N}\left[V^{i}\left(\varepsilon^{i}\right)-\sigma \varepsilon^{i}\right] .
$$

The evolution equation (5) lets $\varepsilon^{i}$ evolve towards local minimizers of $\Phi_{N}$. We are interested in the limit $\nu \rightarrow 0$ which amounts to infinitely fast evolution such that $\varepsilon\left(u_{N}\right)$ attains a local minimizer of $\Phi_{N}$. First we construct the equilibrium points. Inside the $i$-th spring element, the strain must satisfy the condition

$$
\left(V^{i}\right)^{\prime}\left(\varepsilon^{i}\right)=\sigma .
$$

For given total stress $\sigma$, there are at most the three local minimizers (using (A3))

$$
\begin{aligned}
& \breve{\varepsilon}_{a}^{i}(m)=\frac{\sigma}{k_{0}^{i}+\sigma}, \quad \breve{\varepsilon}_{b}^{i}(m)=\frac{\sigma-\sigma_{a}}{k_{b}^{i}}+\varepsilon_{a}^{i}, \\
& \breve{\varepsilon}_{c}^{i}(m)=\frac{\sigma\left(1+\varepsilon_{*}\right)+k_{0}^{i} \varepsilon_{*}}{k_{0}^{i}+\sigma}=\breve{\varepsilon}_{a}^{i}(m)+\varepsilon_{*} .
\end{aligned}
$$

Note that for the derivation of (6), we require that $\delta$ is positive.

In a loading or unloading experiment, the first spring located closer to the bottom of the structure is the softest and yields first, changing its phase, [16]. Next, the second spring yields, and so forth, until the $\beta N$-th spring. (Note that in accordance with (A4), the springs $\beta N+1, \ldots, N$ with small spring constant $\delta$ do not flip.) Therefore, similar to the case of $N$ identical springs, the total state of the series of springs is still completely specified by two scalar parameters $p$ and $q$ and the additional parameter $\beta$. Here, $p, q, 1-p-q$ denote the phase fractions of the minimizers $a, b$, and $c$, which corresponds to having $\beta N p, \beta N q$ 
and $\beta N(1-p-q)$ springs in phase $a, b$, and $c$, respectively. We assumed here that $\beta N p \in \mathbb{N}$.

As $\varepsilon \mapsto V^{i}(\varepsilon)$ is concave in Regime $b$ for all $i \in \mathbb{N}$, if the elongation of a spring in the local minimum $\breve{\varepsilon}_{b}^{i}$ is altered by an arbitrarily small perturbation, it will move (according to the sign of the perturbation) to either $\breve{\varepsilon}_{a}^{i}$ or $\breve{\varepsilon}_{c}^{i}$. As a consequence, any system of $N$ springs with $q \neq 0$ is unstable and we may in the following restrict to the case $q=0$.

The average strain of a system with $\beta N$ springs in equilibrium and the first $\beta N p$ springs in phase a fulfills the identity

$$
\varepsilon(m)=\frac{1}{N} \sum_{i=1}^{\beta N} \breve{\varepsilon}_{a}^{i}(m)+\frac{1}{N} \sum_{i=\beta N p+1}^{\beta N} \varepsilon_{*}+\frac{1}{N} \sum_{i=\beta N+1}^{N} \frac{\sigma}{\delta+\sigma} .
$$

Here we only study the limiting case $\delta \searrow 0$ where there is no further small correction of the damaged springs. In this case, we obtain

$$
\varepsilon(m)=\frac{\beta \sigma(m)}{k_{0}+\sigma(m)}+(1-\beta)+\beta(1-p) \varepsilon_{*}=\frac{\sigma(m)+(1-\beta) k_{0}}{k_{0}+\sigma(m)}+\beta(1-p) \varepsilon_{*},
$$

where we used (A1)-(A4), (3) and (6); especially $\breve{\varepsilon}_{c}^{i}=\breve{\varepsilon}_{a}^{i}+\varepsilon_{*}$.

Resolving (7), we get the stress-strain relationship for a $N$-springs system

$$
\sigma(\varepsilon, m)=\frac{k_{0}\left(\varepsilon-\varepsilon_{p}+\beta-1\right)}{1-\left(\varepsilon-\varepsilon_{p}\right)}
$$

with $\varepsilon_{p}(m):=\beta(1-p) \varepsilon_{*}$. The latter can in a natural way be identified with the plastic strain. From (8) we see that $\sigma$ only depends on $m$ and on the elastic strain $\varepsilon_{\mathrm{el}}:=\varepsilon-\varepsilon_{p}$.

\subsection{Analytic computation of the continuum limit}

We identify the continuum limit of $E_{N}$ in the framework of $\Gamma$-convergence (see, e.g., [4]). This is not a standard procedure as $V$ also depends on the spatial position.

Let $L=1$ (which can always be obtained by rescaling), and $\Omega:=(0,1)$. For prescribed $l>0$, we impose the boundary conditions

$$
u_{0}^{N}=0, u_{N}^{N}=l
$$

This selection of boundary conditions appears natural for the discrete system in the absence of next-to-nearest neighbor-interactions.

We now specify our assumptions on $V$. The mechanical pair-potential is a function of the deformation gradient. In addition, in order to capture the effect of damage expressed in the assumptions (A1)-(A4) above, we need to respect the dependence of $V$ on the spatial position $i$. Let $\operatorname{dom}(V)=[0,1] \times D$ for 
suitable $D \subset \mathbb{R}$ be the domain of definition of $V$. We postulate the following conditions on $V$.

(COND 1) There exist positive constants $c_{1}, C_{1}$, such that

$$
c_{1}(|\varepsilon|-1) \leq V(x, \varepsilon) \leq C_{1}(|\varepsilon|+1) \quad \text { for all }(x, \varepsilon) \in[0,1] \times D .
$$

(COND 2) The function $x \mapsto V(x, \varepsilon)$ is continuous for any $\varepsilon \in D$.

After extending $V$ given by (2) continuously in $i$ by interpolation, we easily verify that this extension (again called $V$ ) satisfies both COND 1 and COND 2. For given deformations $u_{N}:=\left(u_{i}^{N}\right)_{0 \leq i \leq N}$, we may rewrite the overall mechanical energy of the chain as a functional of the discrete displacement gradient,

$$
E_{N}\left(u_{N}\right):=h_{N} \sum_{i=0}^{N-1} V\left(\frac{i}{N}, \frac{u_{i+1}^{N}-u_{i}^{N}}{h_{N}}\right) .
$$

The functional $V$ coincides with (2), but the dependence on the subscript $i$ has moved to the first argument. We scaled $E_{N}$ by $h_{N}$ as we are dealing with a microscopic energy.

Following ideas in [5], we introduce for $N \in \mathbb{N}$ the set $\mathcal{A}_{N}$ of all functions $u: h_{N} \mathbb{Z} \cap[0,1] \rightarrow \mathbb{R}$, setting $u_{i}:=u\left(i h_{N}\right)$. We tacitly identify $\mathcal{A}(0,1)$ with the piecewise affine linear interpolations, i.e.

$$
\mathcal{A}(0,1):=\left\{u:[0,1] \rightarrow \mathbb{R} \mid u \text { is affine in }\left(i h_{N},(i+1) h_{N}\right), 0 \leq i \leq N-1\right\} .
$$

With $u_{i}=u\left(i h_{N}\right)$, as a shorthand notation for the second argument in (10), we introduce the symbol

$$
\nabla^{N} u_{N}(x):=\frac{u_{i+1}^{N}-u_{i}^{N}}{h_{N}}, \quad x \in\left[i h_{N},(i+1) h_{N}\right), 0 \leq i \leq N-1,
$$

which is a discrete approximation of $\nabla u$ with step size $h_{N}$.

For later use we introduce $E_{N}^{l}: \mathcal{A}(0,1) \rightarrow \mathbb{R} \cup\{+\infty\}$ by

$$
E_{N}^{l}(u):= \begin{cases}E_{N}(u), & \text { if } u(0)=0, u(1)=l, \\ +\infty, & \text { otherwise. }\end{cases}
$$

Theorem 1 (Continuum limit of $E_{N}$ )

Let $V$ satisfy the conditions (COND 1), (COND 2) and let $l>0$. Then the functional $E_{N}^{l}$ converges in the $\Gamma$-sense for $N \rightarrow \infty$ to a functional $E^{l}: L^{1}(0,1) \rightarrow \mathbb{R}$ defined by

$$
E^{l}(u):= \begin{cases}\int_{0}^{1} V_{0}^{* *}(x, \nabla u(x)) \mathrm{d} x & \text { if } u \in H^{1,1}(0,1), u(0)=0, u(1)=l, \\ +\infty & \text { else. }\end{cases}
$$

Here, $V_{0}(x, z):=\frac{1}{2} \min \left\{V\left(x, z_{1}\right)+V\left(x, z_{2}\right) \mid z_{1}+z_{2}=2 z\right\}$, and $V_{0}^{* *}$ denotes the convexification of $V_{0}$ (see, e.g., [17]).

Proof (a) Proof of the liminf-inequality. 
Let a sequence $\left(u_{N}\right)_{N \in \mathbb{N}} \subset L^{1}(0,1)$ be given with $u_{N} \rightarrow u$ in $L^{1}(0,1)$ as $N \rightarrow \infty$. W.l.o.g. $E_{N}^{l}\left(u_{N}\right)<\infty$ and thus $E_{N}\left(u_{N}\right)<\infty$. Now, as the bounds in (COND 1) are uniform in $x$, we can apply Theorem 2 in [2] which proofs the liminf-inequality for $\left(u_{N}\right)_{N}$.

(b) Proof of the lim sup-inequality.

Let $u \in L^{1}(0,1)$ be given. We have to show the existence of a sequence $\left(u_{N}\right)_{N \in \mathbb{N}} \subset L^{1}(0,1)$ such that $u_{N} \rightarrow u$ in $L^{1}(0,1)$ as $N \rightarrow \infty$ and

$$
\limsup _{N \rightarrow \infty} E_{N}^{l}\left(u_{N}\right) \leq E^{l}(u) .
$$

By a standard density argument, cf. Part (b3) in the proof of Theorem 2 in [2], we may restrict to the affine case $u(x)=a x+b$.

For the construction we first ignore the boundary conditions (9). Let $N=$ $k m$ for some $m, k \in \mathbb{N}$. We will choose functions $u_{N}$ which are periodic in any subinterval of $(0,1)$ with length $m h_{N}$. We write the integrand as $V\left(d_{N}(x), \cdot\right)$, where $d_{N}:(0,1) \rightarrow \mathbb{R}$ is a piecewise constant bounded function with $d_{N} \rightarrow$ Id for $N \rightarrow \infty$. The method can be generalized to more general situations. With these settings we find

$$
E_{N}\left(u_{N}\right)=\sum_{i=0}^{k-1} \int_{i m h_{N}}^{(i+1) m h_{N}} V\left(d^{i}, \nabla^{N} u_{N}(x)\right) \mathrm{d} x+o(1),
$$

where $d^{i}:=d_{N}\left((i+1 / 2) m h_{N}\right)$; and (COND 2) has been used.

By convexity of $V_{0}^{* *}$ and Carathéodory's theorem (see, e.g., [17]) we know that for any $0 \leq i \leq k-1$, there exist real numbers $\lambda_{1}, \lambda_{2}$ with $0 \leq \lambda_{1}, \lambda_{2} \leq 1$ and $0 \leq \lambda_{1}+\lambda_{2} \leq 1$ such that

$$
\begin{array}{r}
V_{0}^{* *}\left(d^{i}, a\right)=\lambda_{1} V_{0}\left(d^{1, i}, a^{1}\right)+\lambda_{2} V_{0}\left(d^{2, i}, a^{2}\right)+\left(1-\lambda_{1}-\lambda_{2}\right) V_{0}\left(d^{3, i}, a^{3}\right), \\
\left(d^{i}, a\right)=\left(d^{i}, \nabla u\right)=\lambda_{1}\left(d^{i, 1}, a^{1}\right)+\lambda_{2}\left(d^{i, 2}, a^{2}\right)+\left(1-\lambda_{1}-\lambda_{2}\right)\left(d^{i, 3}, a^{3}\right) .
\end{array}
$$

For given $\lambda_{1}, \lambda_{2}$ we introduce the sets

$$
\begin{aligned}
& \Omega_{1}^{N}:=\Omega \cap \cup_{i=0}^{k-1}\left(i h m, i h m+h\left\lfloor\lambda_{1} m\right\rfloor\right], \\
& \Omega_{2}^{N}:=\Omega \cap \cup_{i=0}^{k-1}\left(\left(i h m+h\left\lfloor\lambda_{1} m\right\rfloor\right),\left(i h m+h\left\lfloor\left(\lambda_{1}+\lambda_{2}\right) m\right\rfloor\right],\right. \\
& \Omega_{3}^{N}:=\Omega \cap \cup_{i=0}^{k-1}\left(\left(i h m+h\left\lfloor\left(\lambda_{1}+\lambda_{2}\right) m\right\rfloor\right),(i+1) h m\right\rfloor,
\end{aligned}
$$

such that $\Omega=\Omega_{1}^{N} \cup \Omega_{2}^{N} \cup \Omega_{3}^{N}$.

By definition of $V_{0}$ it holds for $0 \leq i \leq k-1$ and any $s=1,2,3$,

$$
V_{0}\left(d^{s, i}, a^{s}\right)=\frac{1}{2}\left[V\left(d_{1}^{s, i}, a_{1}^{s}\right)+V\left(d_{2}^{s, i}, a_{2}^{s}\right)\right]
$$

where $d^{s, i}, a^{s}, 1 \leq s \leq 3$, are suitable numbers such that $d^{s, i}=\frac{d_{1, i}^{s,}+d_{2}^{s, i}}{2}$ and 
$a^{s}=\frac{a_{1}^{s}+a_{2}^{s}}{2}$. We choose $u_{N}(x)=a_{N}(x) x+b$, where

$$
a_{N}(x)=\left\{\begin{array}{lll}
a_{1}^{1}, & \text { if } \left.x \in \Omega_{1}^{N} \cap \cup_{i=0}^{k-1} \cup_{j=0}^{m / 2-1}\left((i m+2 j) h_{N}, i m+2 j+1\right) h_{N}\right], \\
a_{1}^{2}, & \text { if } x \in \Omega_{2}^{N} \cap \cup_{i=0}^{k-1} \cup_{j=0}^{m / 2-1}\left((i m+2 j) h_{N},(i m+2 j+1) h_{N}\right], \\
a_{1}^{3}, & \text { if } x \in \Omega_{3}^{N} \cap \cup_{i=0}^{k-1} \cup_{j=0}^{m / 2-1}\left((i m+2 j) h_{N},(i m+2 j+1) h_{N}\right], \\
a_{2}^{1}, & \text { if } x \in \Omega_{1}^{N} \cap \cup_{i=0}^{k-1} \cup_{j=0}^{m / 2-1}\left(\left((i m+2 j+1) h_{N},(i m+2 j+2) h_{N}\right],\right. \\
a_{2}^{2}, & \text { if } x \in \Omega_{2}^{N} \cap \cup_{i=0}^{k-1} \cup_{j=0}^{m / 2-1}\left(\left((i m+2 j+1) h_{N},(i m+2 j+2) h_{N}\right],\right. \\
a_{2}^{3}, & \text { if } x \in \Omega_{3}^{N} \cap \cup_{i=0}^{k-1} \cup_{j=0}^{m / 2-1}\left((i m+2 j+1) h_{N},(i m+2 j+2) h_{N}\right] .
\end{array}\right.
$$

For $s=1,2,3$ and $0 \leq i \leq k-1$ we set

$$
d_{N}(x)= \begin{cases}d_{1}^{s, i}, & \text { if } x \in \Omega_{s}^{N} \cap \cup_{j=0}^{m / 2-1}\left((i m+2 j) h_{N},(i m+2 j+1) h_{N}\right], \\ d_{2}^{s, i}, & \text { if } x \in \Omega_{s}^{N} \cap \cup_{j=0}^{m / 2-1}\left((i m+2 j+1) h_{N},(i m+2 j+2) h_{N}\right] .\end{cases}
$$

The ansatz for $d_{N}$ depends on the current interval, as $V$ is $x$-dependent.

Eqn. (11) now reads after setting $\left\lfloor\lambda_{3} m\right\rfloor:=m-\left\lfloor\lambda_{1} m\right\rfloor-\left\lfloor\lambda_{2} m\right\rfloor$ for short

$$
\begin{aligned}
E_{N}\left(u_{N}\right) & =h_{N} \sum_{i=0}^{k-1} \sum_{s=1}^{3}\left\lfloor\lambda_{s} m\right\rfloor \frac{1}{2}\left[V\left(d_{1}^{s, i}, a_{1}^{s}\right)+V\left(d_{2}^{s, i}, a_{2}^{s}\right)\right] \\
& =\sum_{i=0}^{k-1} \sum_{s=1}^{3} \frac{\left\lfloor\lambda_{s} m\right\rfloor}{m} V_{0}\left(d^{s, i}, a^{s}\right) .
\end{aligned}
$$

For $m \rightarrow \infty$ we have $\left\lfloor\lambda_{s} m\right\rfloor / m \rightarrow \lambda_{s}, s=1,2$. Consequently, using (12),

$$
E_{N}\left(u_{N}\right) \rightarrow \int_{0}^{1} V_{0}^{* *}(x, \nabla u(x)) \mathrm{d} x=E^{l}(u) \quad \text { as } N \rightarrow \infty .
$$

We still have to show that $u_{N} \rightarrow u$ in $L^{1}(0,1)$. If in the derivation of Eqn. (14) we formally set $V(x, v): \equiv v$ (which is feasible), we obtain

$$
\int_{0}^{1} \nabla u_{N}(x) \mathrm{d} x=\frac{\left\lfloor\lambda_{1} m\right\rfloor}{m} a^{1}+\frac{\left\lfloor\lambda_{2} m\right\rfloor}{m} a^{2}+\left(1-\frac{\left\lfloor\lambda_{1} m\right\rfloor}{m}-\frac{\left\lfloor\lambda_{2} m\right\rfloor}{m}\right) a^{3}
$$

and in the limit $m \rightarrow \infty$ as above

$$
\lim _{N \rightarrow \infty} \int_{0}^{1} \nabla u_{N}(x) \mathrm{d} x=\lambda_{1} a^{1}+\lambda_{2} a^{2}+\left(1-\lambda_{1}-\lambda_{2}\right) a^{3}=a=\int_{0}^{1} \nabla u(x) \mathrm{d} x,
$$

where Eqn. (13) has been used. By (15), $u_{N} \rightarrow u$ in $L^{r}(0,1)$ for $1 \leq r \leq \infty$.

We still need to incorporate the boundary condition (9). If $u_{N}$ is the recovery sequence from above, we define

$$
v_{N}(x):=\left\{\begin{aligned}
\frac{u_{N}\left(h_{N}\right)}{h_{N}} x, & x \in\left[0, h_{N}\right), \\
u_{N}(x), & x \in\left[h_{N}, 1-h_{N}\right], \\
\frac{l-u_{N}\left(1-h_{N}\right)}{h_{N}}(x-1)+l, & x \in\left(1-h_{N}, 1\right] .
\end{aligned}\right.
$$

By construction, $v_{N}$ is continuous on $[0,1]$ with $v_{N}(0)=0, v_{N}(1)=l$, and $\lim _{N \rightarrow \infty}\left(E_{N}\left(u_{N}\right)-E_{N}\left(v_{N}\right)\right)=0$, which shows that $v_{N}$ is the sought recovery sequence. 


\section{$3 \quad$ Numerical results}

We study in this section the numerical convergence of the stress-strain response of finite mass-spring systems to the continuum limit of Eqn. (8). We analyze the overall loading-unloading response of discrete systems composed of $N=x+y$ springs, where $x$ denotes the number of undamaged springs, while $y$ specifies the number of damaged springs. For the spring constants, we use the parameters $k_{0}=50.00 \times 10^{6} \mathrm{~Pa}, k_{b}=-22.44 \times 10^{6} \mathrm{~Pa}, \varepsilon_{a}=0.25, \varepsilon_{*}=0.52, \delta=50.00 \times 10^{2}$ $\mathrm{Pa}$, which correspond to $\sigma_{a}=16.67 \mathrm{MPa}, \sigma_{c}=5.00 \mathrm{MPa}$, and $\Delta \sigma=\sigma_{c}-\sigma_{a}=$ $-11.67 \mathrm{MPa}$.
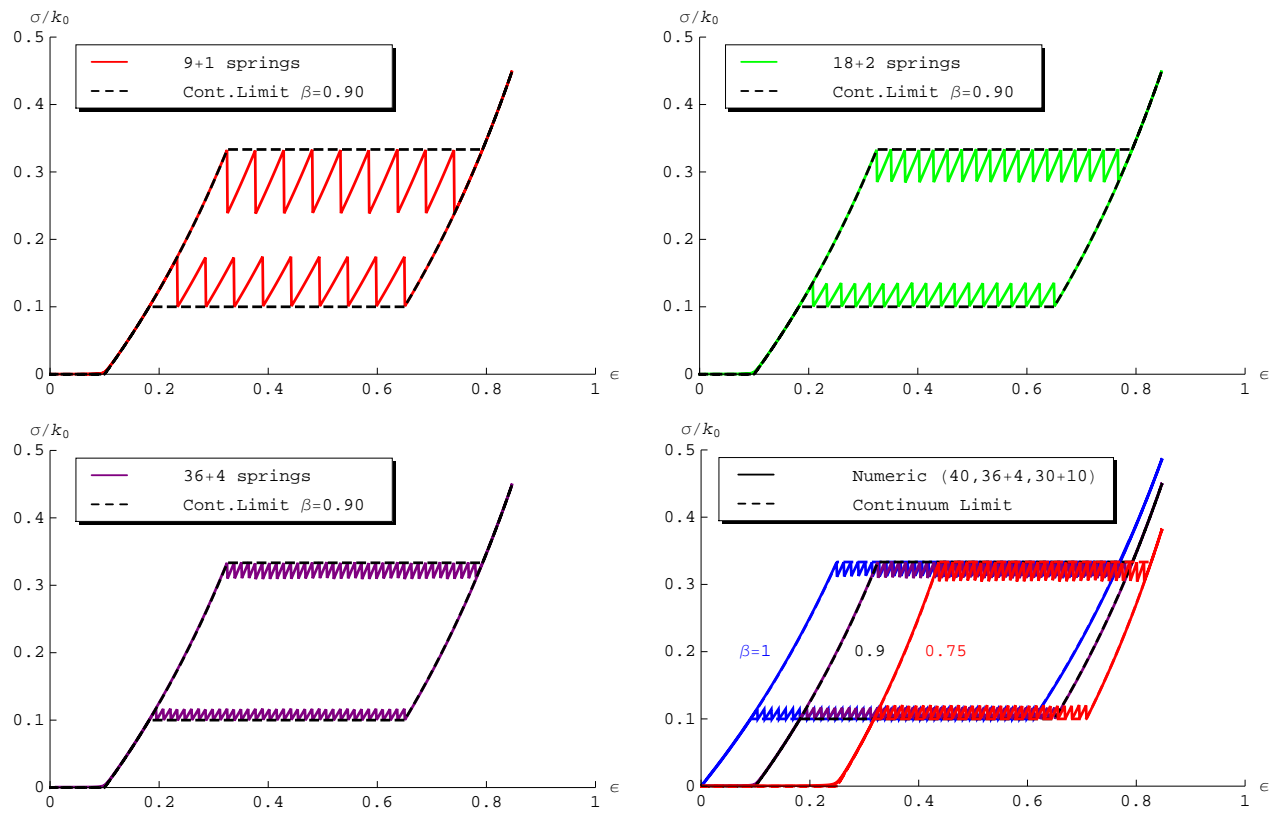

Figure 1: Numerical overall stress-strain response of bistable mass-spring chains for different total number of springs $N$.

Fig. 1 shows the results for different $x, y$. The stress-strain curves of the discrete microscopic chains follow sawtooth patterns which alternate elastic and plastic steps. The plastic steps are characterized by fixed total strain and microscopic branch switching. Such sawtooth-like responses converge to a perfectly plastic behavior with a loading $\left(\sigma=\sigma_{a}\right)$ and an unloading plateau $\left(\sigma=\sigma_{c}\right)$ for increasing values of $N$, as predicted by Eqn. (8). It is worth observing that the stress is zero for $\varepsilon \leq 1-\beta$, while for $\varepsilon>1-\beta$ that the system is able to bear stresses $\sigma>0$. Fig. 1 shows, in addition, that the energy dissipation capacity of the system (area enclosed by stress-strain curve in a loading-unloading cycle) reduces for decreasing $\beta$, that is for increasing number of damaged springs (bottom-right panel). Remarkably, the quantity $1-\beta$ can be regarded as the 'activation' strain of the system. 


\section{Concluding remarks}

In this paper, for the first time a bistable-spring ansatz has been proposed capable of incorporating damage in plastically deformed materials.

We have shown that a suitable modification of the model recently proposed by [9] for CNT foams is able to handle preconditioning induced material damage, which is characterized by an activation strain different from zero; a reduction in the energy dissipation capacity; and permanent deformation. The latter, in particular, coincides with the activation strain. The new model allows us to extend the 'transformational plasticity' concept discussed by [15] from timeindependent hysteretic behavior to fatigue-type material damage. It applies to a wide class of materials showing Mullins-like behavior, [13], which includes besides CNT arrays rubber-like and soft biological materials.

We address a multiscale formulation of the present model, accounting for graded mechanical properties along the height of the structure, [16], and the analytic computation of the energy dissipated by the system in the continuum limit in future work.

\section{Acknowledgements}

FF acknowledges the support of the Italian Network of Seismic Engineering Laboratories (ReLUIS) and the Italian Civil Protection Department (DPC), through the ReLUIS-DPC grant 2010/2013. TB acknowledges the support of the German Research Community (DFG) through grant BL 512 4/1. JRR gratefully acknowledges the U.S. Department of Defense and the Army Research Office for their support via a National Defense Science \& Engineering Graduate (NDSEG) fellowship. CD acknowledges support from the Institute for Collaborative Biotechnologies under contract W911NF-09-D-0001 with the Army Research Office.

\section{References}

[1] Baughman, R.H., Zakidov, A.A., and de Heer, W.A.(2002). Carbon nanotubes-the route toward application, Science, 297(5582):787-792

[2] Blesgen, T.(2007). The competition of elastic energy and surface energy in discrete numerical systems. Advances in Comput. Mathematics, 27:179194.

[3] Bradford, P.D., Wang, X., Zhao, H., and T.Z.Y.(2011). Tuning the compressive mechanical properties of carbon nanotube foam. Carbon, 49:28342841.

[4] Braides A.(2002) Gamma-convergence for beginners, Oxford Lecture Series in Mathematics, Oxford University Press 
[5] Braides, A., Dal Maso, G., and Garroni, A.(1999). Variational formulation of softening phenomena in fracture mechanics: the one-dimensional case. Arch. Rat. Mech. Anal., 146:23-58

[6] Cao, A., Dickrell, P., Sawyer, W.G., Ghasemi-Neihad, M., Ajayan, P.(2005). Super-compressible foamlike carbon nanotube films. Science, 310:130\%-1310.

[7] De Tommasi, D. and Puglisi, G.(2006). A micromechanics-based model for the mullins effect. J. Rheol., 50(4):495-512

[8] Dorfmann, A. and Ogden, R.W.(2004). A constitutive model for the Mullins effect with permanent set in particle-reinforced rubber. Int. J. Solids Struct., 41:1855-1878.

[9] Fraternali, F., Blesgen, T., Amendola, A., Daraio C.(2010). Multiscale mass-spring models of carbon nanotube foams. J. Mech. Phys. Solid., 59(1):89-102.

[10] Gibson, L.J. and Ashby, M.F.(1999) Cellular Solids: Structure and Property. Pergamon Press, Oxford, 2nd edition.

[11] Hutchens, S.B., Hall, L., and Greer, J.R.(2010). In situ mechanical testing reveals periodic buckle nucleation and propagation in carbon nanotube bundles. Adv. Funct. Mater., 20:1-9.

[12] Misra, A., Greer, J., and Daraio, C.(2009). Strain rate effects in the mechanical response of polymer anchored carbon nanotube foams. Advanced Materials, 21:334-338.

[13] Mullins, L.(1947). Effect of stretching on the properties of rubber. J. Rubber Res., 16(12):275-289.

[14] Puglisi, G. and Truskinovsky, L.(2002). Rate-dependent hysteresis in a bistable chain. J. Mech. Phys. Solids, 50(2):165-18\%.

[15] Puglisi, G. and Truskinovsky, L. (2005). Thermodynamics of rate-independent plasticity. J. Mech. Phys. Solids, 53(3):655-679.

[16] Raney, J.R., Fraternali, F., Amendola, A., and Daraio, C.(2011). Modeling and in situ identification of material parameters for layered structures based on carbon nanotube arrays. Compos. Struct., 93(11):3013-3018.

[17] Rockafellar, R.(1997). Convex Analysis, 2.Edition. Princeton University Press.

[18] Yaglioglu, O.(2007) Carbon Nanotube Based Electromechanical Probes. $\mathrm{PhD}$ thesis, Massachesetts Institute of Technology, Cambridge, Massachusetts. 